Europhysics Letters

PREPRINT

\title{
Quantum Irreversibility of Energy Spreading
}

\author{
Tsampikos Kottos $^{1}$, And Doron Cohen ${ }^{2}$ \\ 1 Max-Planck-Institut für Strömungsforschung, Bunsenstraße 10, D-37073 Göttingen, Germany \\ 2 Department of Physics, Ben-Gurion University, Beer-Sheva 84105, Israel
}

PACS. 03.65.-w - Quantum mechanics.

PACS. 05.45.Mt - Quantum chaos.

PACS. 73.23.-b - Mesoscopic systems.

\begin{abstract}
The analysis of dissipation and dephasing in driven mesoscopic devices requires a distinction between two notions of quantum irreversibility. One ("Loschmidt echo") is related to "time reversal", while the other is related to "driving reversal". In the latter context we define the time of maximum return ("compensation time") which generalizes the notion of "echo time". Non-perturbative features manifest themselves in the energy spreading process. This is demonstrated for the prototype random-matrix Wigner model, where the compensation time and the system response exhibit a non-universal scaling behavior.
\end{abstract}

Quantum Irreversibility (QI) [1] is a subject of a recent intensive research activity due to its relevance to quantum computing [2]. It turns out that the analysis of dissipation and dephasing in driven mesoscopic devices requires a distinction between two different notions of "irreversibility". One is based on the "piston model" paradigm (PMP), while the other is based on the "ice cube in a cup of hot water" paradigm (ICP). The latter notion has been adopted by the recent literature 1, 2, and it is related to the studies of dephasing due to the interaction with chaotic degrees of freedom 3. The PMP on the other hand has direct relevance to our recent studies [4] of the (non-perturbative) response of driven chaotic mesoscopic systems.

In the PMP case we say that a process is reversible if it is possible to "undo" it. Consider a gas inside a cylinder with a piston. Let us shift the piston inside. Due to the compression the gas is heated up. Can we undo the "heating" simply by shifting the piston outside, back to its original position? If the answer is "yes", as in the case of strictly adiabatic process, then the process is said to be reversible. In the ICP case we consider the melting process of an ice cube. Let us assume that after some time we reverse the velocities of all the molecules. If the external conditions are kept strictly the same, we expect the ice-cube to re-emerge out of the water. In practice the external conditions (fields) are not exactly the same, and as a result we have what looks like irreversibility.

It is also essential to define precisely what is the measure for QI. The prevailing possibility is to take the survival probability as a measure [1]. Another possibility is to take the energy spreading as a measure. (The energy spreading is defined as the square root of the energy variance.) The latter definition goes well with the PMP, and it has a well defined classical limit.

(c) EDP Sciences 
Thus the ICP is related to "time reversal" experiments, while the PMP is related to "driving reversal" experiments. Later we are going to put the focus on PMP irreversibility. A thorough understanding of the one-period driving reversal scenario is both important by itself, and also constitutes a bridge towards a theory for the response to periodic driving [4. It should be realized that irreversibility in the PMP sense implies irreversible diffusion in energy space. This diffusion leads to irreversible energy absorption ("dissipation") in periodically driven mesoscopic devices 5. One purpose of this Letter is to sharpen the notion "irreversible diffusion".

The structure of this Letter is as follows: First we discuss the mathematical formulation of the ICP and the PMP. Then we focus on QI in the PMP sense, which has not been done in the past as far as we know. In this context we define the time of maximum return ("compensation time"), which constitutes a generalization of the notion of "echo time". The specific model that we study is the well known Wigner model 6]. We shall explain that this is the simplest model that contains the main ingredients of a physical problem. We define the notions of perturbative/non-perturbative regimes and in particular we explain the adiabatic/sudden limits. The nature of QI in the different dynamical regimes is found to be distinct. The theoretical considerations are supported by the analysis of extensive numerical simulations. The new insights regarding the analysis of wavepacket dynamics in energy space 8] are summarized in the last paragraph.

Consider a system whose evolution is governed by the chaotic Hamiltonian $\mathcal{H}=\mathcal{H}\left(Q_{\alpha}, P_{\alpha} ; x\right)$, where $\left(Q_{\alpha}, P_{\alpha}\right)$ is a set of canonical coordinates, and $x=x(t)$ with $0<t<T / 2$ is a time dependent parameter. The corresponding evolution operator will be denoted by $U[x]$. In the ICP case the parameter $x$ represents the external fields. The physical conditions that correspond to the "forward" evolution are represented by $x=x_{\mathrm{A}}(t)$, while the physical conditions that correspond to the "backward" evolution are the time-reversal of $x=x_{\mathrm{B}}(t)$. If $x_{\mathrm{B}}(t)=x_{\mathrm{A}}(t)$ then we have complete reversibility, otherwise we can define a generalized fidelity amplitude as $F(T)=\left\langle\Psi_{0}\left|U\left[x_{\mathrm{B}}\right]^{-1} U\left[x_{\mathrm{A}}\right]\right| \Psi_{0}\right\rangle$, where $\Psi_{0}$ is the initial preparation. The generalized fidelity amplitude $F(T)$ is in fact [3] the Feynman-Vernon influence functional, and $|F(t)| \leq 1$ is the so called dephasing factor. For constant $x_{\mathrm{A}}$ and $x_{\mathrm{B}}$ we can write $U\left[x_{\mathrm{A}}\right]=\exp \left(-i T \mathcal{H}_{\mathrm{A}} / 2 \hbar\right)$ and $U\left[x_{\mathrm{B}}\right]=\exp \left(-i T \mathcal{H}_{\mathrm{B}} / 2 \hbar\right)$, leading to the currently prevailing definition of the fidelity, also know as Loschmidt echo, as a measure for quantum irreversibility [1.

To be more specific we assume that the variations of the external fields are classically small, so that we can linearize the Hamiltonian as follows: $\mathcal{H}=\mathcal{H}_{0}+x \mathcal{W}$. Then, for constant perturbation $x= \pm \epsilon$, we can write $\mathcal{H}_{\mathrm{A}}=\mathcal{H}_{0}+\epsilon \mathcal{W}$ and $\mathcal{H}_{\mathrm{B}}=\mathcal{H}_{0}-\epsilon \mathcal{W}$, where $\epsilon$ is the amplitude of the perturbation. Note that the prevailing definition of the fidelity $\mathcal{P}_{\mathrm{LE}}(T)=|F(T)|^{2}$, also known as Loschmidt echo, takes the form

$$
\mathcal{P}_{\mathrm{LE}}(T)=\left|\left\langle\Psi_{0}\left|\mathrm{e}^{-i \frac{T}{2 \hbar}\left(-\mathcal{H}_{0}+\epsilon \mathcal{W}\right)} \mathrm{e}^{-i \frac{T}{2 \hbar}\left(\mathcal{H}_{0}+\epsilon \mathcal{W}\right)}\right| \Psi_{0}\right\rangle\right|^{2}
$$

In complete analogy with the ICP, we can define $\mathcal{P}(T)=\left|\left\langle\Psi_{0}\left|U\left[x_{\mathrm{B}}(\mathrm{rev})\right] U\left[x_{\mathrm{A}}\right]\right| \Psi_{0}\right\rangle\right|^{2}$. Here $x_{\mathrm{B}}(\mathrm{rev})$ corresponds to the reversed driving process, eg pushing the piston back to its initial location. For constant $x_{\mathrm{A}}$ and $x_{\mathrm{B}}$ we can write $U\left[x_{\mathrm{A}}\right]=\exp \left(-i \frac{T}{2} \mathcal{H}_{\mathrm{A}}\right)$ and $U\left[x_{\mathrm{B}}(\mathrm{rev})\right]=$ $\exp \left(-i \frac{T}{2} \mathcal{H}_{\mathrm{B}}\right)$, and for the linearized Hamiltonian we get

$$
\mathcal{P}(T)=\left|\left\langle\Psi_{0}\left|\mathrm{e}^{-i \frac{T}{2 \hbar}\left(\mathcal{H}_{0}-\epsilon \mathcal{W}\right)} \mathrm{e}^{-i \frac{T}{2 \hbar}\left(\mathcal{H}_{0}+\epsilon \mathcal{W}\right)}\right| \Psi_{0}\right\rangle\right|^{2}
$$

If we take the PMP literally, then the parameter $\epsilon$ represents either a small displacement of a piston, or its velocity. The latter interpretation emerges if we write the Schrödinger equation in the adiabatic basis (See eg Eq. (55) of Ref. [5]). Similarly, in case of an Aharonov-Bohm 
ring system, the parameter $\epsilon$ represents either the magnetic flux through a ring, or else it may represent the electro-motive-force (measured in Volts). The latter, by Faraday law, is the time derivative of the former. As a matter of terminology, note that if $\epsilon$ is interpreted as "velocity", then what we call below "standard perturbative regime" is in fact the "adiabatic regime" (small $\epsilon)$. Later we also discuss the notion of "sudden regime" (large $\epsilon$ ).

At first sight the two definitions of $\mathcal{P}(T)$ look very similar: it is as if the role of $\mathcal{H}_{0}$ and $\mathcal{W}$ is interchanged. Also one may (wrongly) think that there is some kind of symmetry between the "adiabatic limit" $(\epsilon \rightarrow 0)$, and the opposing "sudden limit" $(\epsilon \rightarrow \infty)$. This superficial "algebraic" symmetry of the definitions is misleading. One should be aware that in typical circumstances $\mathcal{H}_{0}$ has an unbounded spectrum, unlike the perturbation $\mathcal{W}$. Specifically we refer here to quantized chaotic systems. In the standard representation $\mathcal{H}_{0}=\mathbf{E}$ is a diagonal matrix whose elements are the ordered energies $\left\{E_{n}\right\}$. We limit our interest to a classically small (but quantum mechanically large) energy interval where the mean level spacing $\Delta$ is roughly constant. Assuming that the classical motion is characterized by a finite correlation time $\tau_{c l}$, it follows that the matrix $\mathcal{W}=\mathbf{B}$ in the standard representation is a banded matrix which looks random 7 . Its bandwidth is $\Delta=2 \pi \hbar / \tau_{c l}$. The simplest model that captures this generic feature is the Wigner banded random matrix model (BRM) 6]. The non-vanishing couplings are within the band $0<|n-m| \leq b$. These coupling elements are zero on the average, and they are characterized by the variance $\sigma=\left(\left\langle\left|\mathbf{B}_{n m}\right|^{2}\right\rangle\right)^{1 / 2}$. For sake of later convenience we define the bandwidth in energy units as $\Delta_{b}=b \Delta$, and describe the band profile using a spectral function $\tilde{C}(\omega)$ that equals $2 \pi \hbar \sigma^{2} / \Delta$ for $|\hbar \omega|<\Delta_{b}$, and zero otherwise. This is in fact the Fourier transform of $C(\tau)=2 b \sigma^{2} \sin \left(\tau / \tau_{c l}\right) /\left(\tau / \tau_{c l}\right)$. The function $C(\tau)$ describes the time autocorrelation of $\mathcal{W}$ in the Heisenberg picture.

We are interested in the time evolution of the energy distribution. The corresponding time-dependent Schrödinger equation has been integrated numerically using the self-expanding algorithm of 8 ] to eliminate finite-size effects (the remarkable accuracy is demonstrated in Fig. 1a). In all the simulations the initial state $\left|\Psi_{0}\right\rangle$ is assumed to be an eigenstate $|m\rangle$ of $\mathcal{H}_{0}$. In the first half period $(t<T / 2)$ we have "conventional" wavepacket dynamics that we had studied in [8]. What we want to discuss in this Letter is the dynamics for $t>T / 2$, after the driving is reversed. The probability distribution after time $t$ is $P_{t}(n \mid m)=|\langle n|U(t)| m\rangle|^{2}$. Averaging over realizations of the Hamiltonian one obtains the average profile $P_{t}(n-m)$. We characterize the evolving distribution using two different measures: The survival probability $\mathcal{P}(t)=\mathcal{P}(t ; T)=P_{t}(r=0)$, and the energy spreading $\delta E(t)=\delta E(t ; T)=\Delta \times \sqrt{\sum_{r} r^{2} P_{t}(r)}$. Thus the energy distribution at the end of the driving cycle is characterized by $\mathcal{P}(T)=\mathcal{P}(T ; T)$ and $\delta E(T)=\delta E(T ; T)$.

Looking on Fig. 1b and Fig. 1c we see that it is natural to define a time $t_{r}$ of maximal return. This is the time, after the driving reversal, when we observe maximum compensation. The "compensation time" $\left(t_{r}\right)$ is determined either by the minimum of the spreading $\delta E(t)$ or by the minimum of the total transition probability $p(t)=(1-\mathcal{P}(t))$. It is clear, by simple inspection of the plots in Fig. 1, that the time of "maximal return" is in general not $t_{r}=T$ but rather $(T / 2) \leq t_{r} \leq T$. Therefore we cannot regard this compensation as an "echo" phenomena (the term "echo" implies maximal return at $t_{r}=T$ ). The dependence of $t_{r}$ on $T$ for various values of $\epsilon$ is presented in Fig.2a and Fig.2b.

We want to understand the effect of driving reversal on the spreading/decay processes, and in particular to determine whether (or to what degree) the spreading/decay processes are reversible. The systematic strategy for the analysis of the dynamics consists of two stages: The first stage is to define the different $\epsilon$ regimes in the corresponding parametric problem. As far as this Letter is concerned 9 the important distinction is between the standard perturbative regime $(\epsilon<\Delta / \sigma)$, the Wigner Lorentzian regime $\left(\Delta / \sigma<\epsilon<b^{1 / 2} \Delta / \sigma\right)$, and the non- 
perturbative semicircle regime $\left(\epsilon>b^{1 / 2} \Delta / \sigma\right)$. The second stage is to consider the time dependent scenario separately in each regime. The driving is characterized by two parameters: One is the amplitude $\epsilon$, and the other is the period $T$. Thus we have a two dimensional parameter space $(T, \epsilon)$ to study, in analogy with having $(\Omega, A)$ space in case of periodic driving [4. If the perturbation parameter $\epsilon$ has the meaning of "velocity", then the "standard perturbative regime" is in fact the "adiabatic regime". By association one naturally wonders whether there is also a "sudden regime". We shall come to this issue later on.

It is natural to inquire whether perturbation theory is applicable for the analysis of the driving reversal scenario. Using first order perturbation theory one obtains the following results for either the total transition probability $p(t)=(1-\mathcal{P}(t))$ or the energy spreading $(\delta E(t))^{2}$ :

$$
\epsilon^{2} \times \int_{-\infty}^{\infty} \frac{d \omega}{2 \pi}\left[\frac{1}{(\hbar \omega)^{2}}\right] \tilde{F}_{t}(\omega) \tilde{C}(\omega)
$$

where the expression in the square brackets should be excluded in case of $\delta E(t)$. The spectral content of the driving is $\tilde{F}_{t}(\omega)=2(1-\cos (\omega t))$ for $t<T / 2$, and $\tilde{F}_{t}(\omega)=\left|1-2 \mathrm{e}^{i \omega T / 2}+\mathrm{e}^{i \omega t}\right|$ otherwise. In Fig. 1b we display the rescaled energy spreading $\delta E(t) / \epsilon$ for a representative period $T$ and various $\epsilon$. For small $\epsilon$ we observe the scaling which is implied by Eq. (3). But for larger $\epsilon$ we observe a different (non-perturbative) behavior. Similar results (Fig. 1c) are found in case of $p(t) / \epsilon^{2}$.

Before we go on in explaining the new "physics" in the non-perturbative regime, we would like to give, for sake of completeness, a very concise summary of the "physics" in the perturbative regime. The following is based on further analysis of Eq.(3), extending the approach of Ref. [8]. In the "standard perturbative" (or "adiabatic") regime, the spreading probability remains concentrated all the time in the initial level. In the "Wigner Lorentzian regime" the energy spreading profile is characterized by two energy scales $\Delta_{b}$ and $\Gamma=2 \pi(\sigma \epsilon)^{2} / \Delta$. If we are deep in this regime we have $1 \ll \Gamma \ll \Delta_{b}$. The energy scale $\Gamma$ is important for $\mathcal{P}(t)$, because this is the energy range where most of the probability is concentrated (the "core" component). In contrast to that the energy scale that is important for $\delta E(t)$ is $\Delta_{b}$, because the variance is determined by the perturbative tails of the energy distribution, and not by the width of the non-perturbative "core" component. This means that in the Wigner Lorentzian regime the "physics" of reversibility is different, depending whether we look on $\mathcal{P}(t)$ or on $\delta E(t)$. To be specific, for $\delta E(t)$ we have reversibility provided $t<\tau_{c l}$, while for $\mathcal{P}(t)$ we have reversibility for $t<\hbar / \Gamma$.

We turn now to discuss the dynamics in the non-perturbative regime, which is our main interest. In the absence of driving reversal [8] we get diffusion $(\delta E(t) \propto \sqrt{t})$ for $t>t_{\mathrm{prt}}(\epsilon)$, where

$$
t_{\mathrm{prt}}(\epsilon)=\hbar /(\sqrt{b} \sigma \epsilon)
$$

If $T / 2<t_{\mathrm{prt}}(\epsilon)$, this non-perturbative diffusion does not have a chance to develop, and therefore we can still trust Eq. (33). So the interesting case is $T / 2>t_{\mathrm{prt}}(\epsilon)$, which means large enough $\epsilon$. It is extremely important to realize that without reversing the driving, the presence or the absence of $\mathbf{E}$ cannot be detected. It is only by driving reversal that we can easily determine (as in Fig. 1a) whether the diffusion process is reversible or irreversible.

In order to analyze irreversibility we should distinguish between two stages in the nonperturbative diffusion process. The first stage $\left(t_{\mathrm{prt}}<t<t_{\mathrm{sdn}}\right)$ is reversible, while the second stage $\left(t>t_{\mathrm{sdn}}\right)$ is irreversible. [For much longer time scales we have recurrences or localization which are not the issue of this Letter [9].] The second time scale $\left(t_{\mathrm{sdn}}\right)$ is "new". It did 
not appear in our "wavepacket dynamics" study [8], because it can be detected only by time/driving reversal experiment.

We can determine the time scale $t_{\mathrm{sdn}}$ as follows. The diffusion coefficient is $D_{\mathrm{E}}=$ $\Delta^{2} b^{5 / 2} \sigma \epsilon / \hbar$ up to a numerical prefactor [8]. The diffusion law is $\delta E^{2}(t)=D_{\mathrm{E}} t$. The diffusion process is reversible as long as $\mathbf{E}$ does not affects the relative phases of the participating energy levels. This means that the condition for reversibility is $(\delta E(t) \times t) / \hbar \ll 1$. The latter inequality can be written as $t<t_{\mathrm{sdn}}(\epsilon)$, where

$$
t_{\mathrm{sdn}}(\epsilon)=\left(\frac{\hbar^{2}}{D_{\mathrm{E}}}\right)^{1 / 3}=\left(\frac{\hbar^{3}}{\Delta^{2} b^{5 / 2} \sigma \epsilon}\right)^{1 / 3} .
$$

This considerations imply that in the non-perturbative regime we should have non-universal scaling with respect to $\epsilon^{1 / 3} \times T$. This obviously goes beyond any implications of perturbation theory (Eq.(3) ). The numerical analysis (Fig. 2) confirms the above claim.

The non-perturbative diffusion process involves only one energy scale, and therefore in the non-perturbative regime the scaling of $\delta E(T)$ and $\mathcal{P}(T)$ is similar (Fig. 3). The non universal scaling of $\mathcal{P}(T)$ versus $\epsilon^{1 / 3} \times T$ is very different from the observed behavior in the Wigner regime (not shown) where the scaling is versus $\epsilon^{2} \times T$. Also for $\delta E(T)$ we observe the same non-perturbative scaling by plotting $\delta E(T) / \epsilon^{1 / 3}$ versus $\epsilon^{1 / 3} \times T$. The latter should be contrasted with the perturbative $\epsilon^{0} \times T$ scaling which is observed in the Wigner regime (not shown). The scaling of the vertical axis is explained on the basis of diffusion process after $t_{r}$, which is of the same nature as for $t<T / 2$.

We are now able to identify the "sudden regime". This is the $(T, \epsilon)$ regime which is defined by the condition $T \ll t_{\mathrm{sdn}}(\epsilon)$. The condition is never satisfied in the $\epsilon \rightarrow \infty$ limit. If we interpret $\epsilon$ as "velocity" then this looks quite strange in first sight, because we are used to associate the sudden limit with having a very "fast" process. The subtlety is resolved once we realize that in a proper textbook formulation of the sudden limit we should take $\epsilon \rightarrow \infty$, while holding the "displacement" $(\epsilon \times T)$ equal to a constant value.

In conclusion of the analysis of non-perturbative features of QI in the PMP sense for Wigner model, we would like to emphasize the distinction between "reversible" and "irreversible" diffusion, and the associated identification of the "sudden regime".

\section{REFERENCES}

[1] A. Peres, Phys. Rev. A 30, 1610 (1984).

[2] R.A. Jalabert and H.M. Pastawski, Phys. Rev. Lett. 86, 2490 (2001); F.M. Cucchietti, H.M. Pastawski, R. Jalabert Physica A, 283, 285 (2000); F.M. Cucchietti, H.M. Pastawski and D.A. Wisniacki, Phys. Rev. E 65, 045206 (2002); Ph. Jacquod, P.G. Silvestrov and C.W.J. Beenakker, Phys. Rev. E 64, 055203 (2001); N.R. Cerruti and S. Tomsovic, Phys. Rev. Lett. 88, 054103 (2002); T. Prosen, quant-ph/0106149 Z.P. Karkuszewski, C. Jarzynski, and W.H. Zurek, Phys. Rev. Lett. 89, 170405 (2002). D.A. Wisniacki and D. Cohen, Phys. Rev. E 66, 046209 (2002).

[3] D. Cohen, Phys. Rev. E 65, 026218 (2002). This publication is regarding dephasing due to the interaction with chaotic degrees of freedom. The relation to the QI studies of Ref. 2] is explained there. Note that main stream literature is dealing mainly with dephasing due to the interaction with bath degrees of freedom. See for example Quantum Dissipative Systems, U. Weiss, World Scientific, Singapore (1999); Quantum Transport and Dissipation, T. Dittrich, P. Hnggi, G. L. Ingold, B. Kramer, G. Schn, and W. Zwerger, Wiley-VCH, Weinheim (1998); W. Zurek, Phys. Scripta 76, 186 (1998). Interaction with RMT bath is discussed by P. A. Bulgac, G.D. Dang and D. Kusnezov, Phys. Rev. E 58, 196 (1998). Some recent related publications are: D. Braun, F. Haake, and W.T. Strunz, Phys. Rev. Lett. 86, 2913 (2001); T. Gorin, T.H. Seligman, J. of Optics B 4, S386 (2002). 
[4] D. Cohen and T. Kottos, Phys. Rev. Lett. 85, 4839 (2000).

[5] D. Cohen, Annals of Physics 283, 175 (2000); D. Cohen in "Proceedings of the International School of Physics Enrico Fermi Course CXLIII", Edited by G. Casati, I. Guarneri and U. Smilansky, IOS Press, Amsterdam (2000). Y.V.Fyodorov and A.D.Mirlin, Phys. Rev. Lett. 67, 2405 (1991).

[6] E. Wigner, Ann. Math 62548 (1955); 65203 (1957); V.V. Flambaum, A.A. Gribakina, G.F. Gribakin and M.G. Kozlov, Phys. Rev. A 50267 (1994); G. Casati, B.V. Chirikov, I. Guarneri, F.M. Izrailev, Phys. Rev. E 48, R1613 (1993); Phys. Lett. A 223, 430 (1996); Ph. Jacquod and D. L. Shepelyansky, Phys. Rev. Lett. 75, 3501 (1995); Y. V. Fyodorov, O. A. Chubykalo, F. M. Izrailev, and G. Casati, ibid. 76, 1603 (1996).

[7] M. Feingold and A. Peres, Phys. Rev. A 34 591, (1986); M. Feingold, D. Leitner, M. Wilkinson, Phys. Rev. Lett. 66, 986 (1991); M. Feingold, A. Gioletta, F. M. Izrailev, L. Molinari, ibid. 70, 2936 (1993); M. Wilkinson, M. Feingold, D. Leitner, J. Phys. A 24, 175 (1991).

[8] D. Cohen, F. Izrailev and T. Kottos, Phys. Rev. Lett., 842052 (2000); T. Kottos and D. Cohen, Phys. Rev. E 64, R-065202 (2001).

[9] Long time recurrences are not an issue of the present Letter. This is the reason why we do not make a distinction between the "ergodic" and the "localization" regimes. See [8] for details.

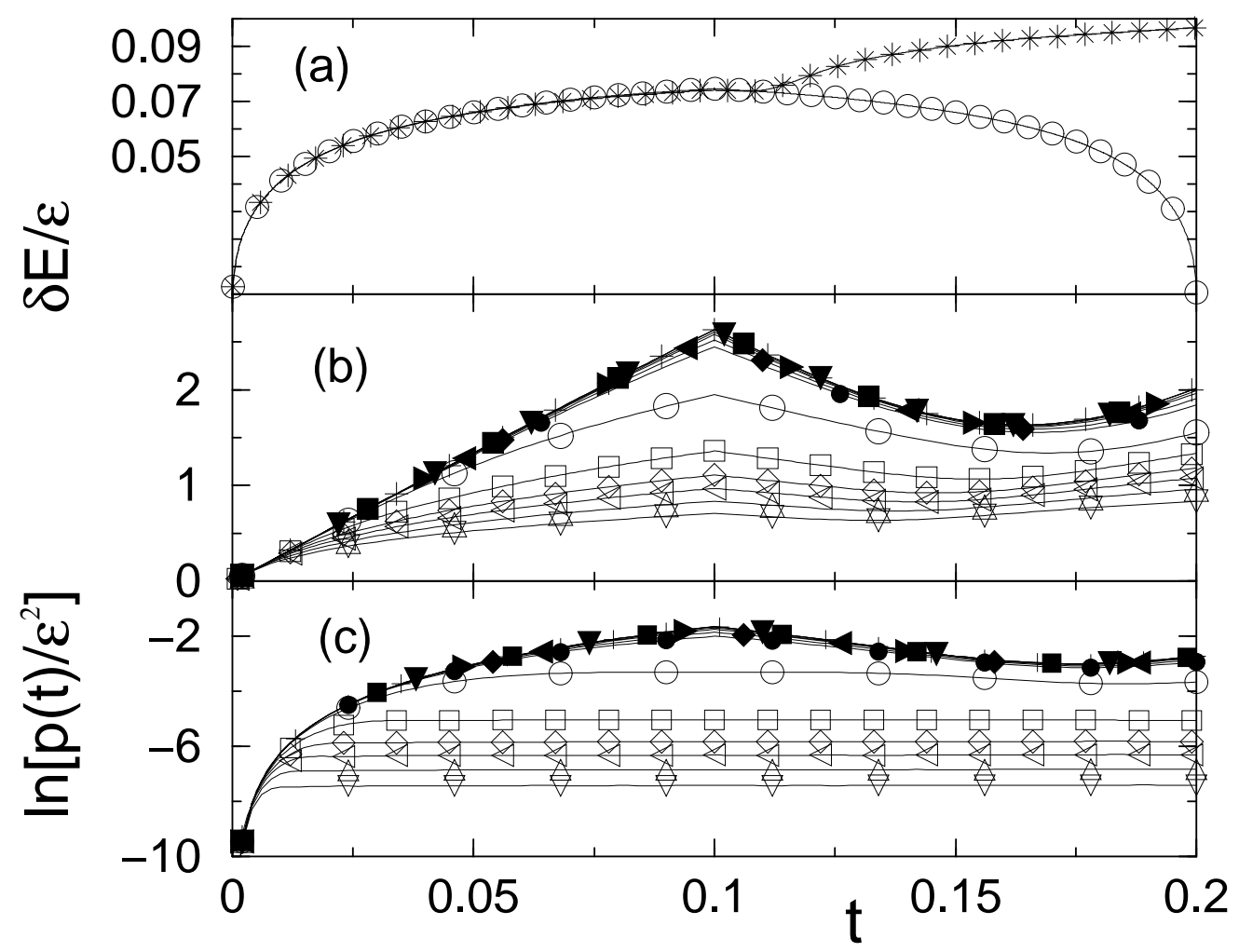

FIG.1: Energy spreading and total transition probability as a function of time. We use units such that $\Delta=\sigma=\hbar=1$, and $b=10$. Typically we take an average over more than 100 different realizations in each run. (a) The actual evolution $(\star)$ with $\mathbf{E} \neq 0$ is compared with the evolution that would be obtained (o) if we had $\mathbf{E}=0$. The simulation is done for an extremely large perturbation $(\epsilon=1000)$. The remarkable reversibility in the $\mathbf{E}=0$ case is an indication for the accuracy of the numerical simulations. (b) The rescaled energy spreading $\delta E(t) / \epsilon$, for $T=0.2$, and for $\epsilon \leq 40$. The clustering of curves corresponds to $\epsilon \leq 2.5$. (c) The same for the rescaled total transition probability $p(t) / \epsilon^{2}$. 


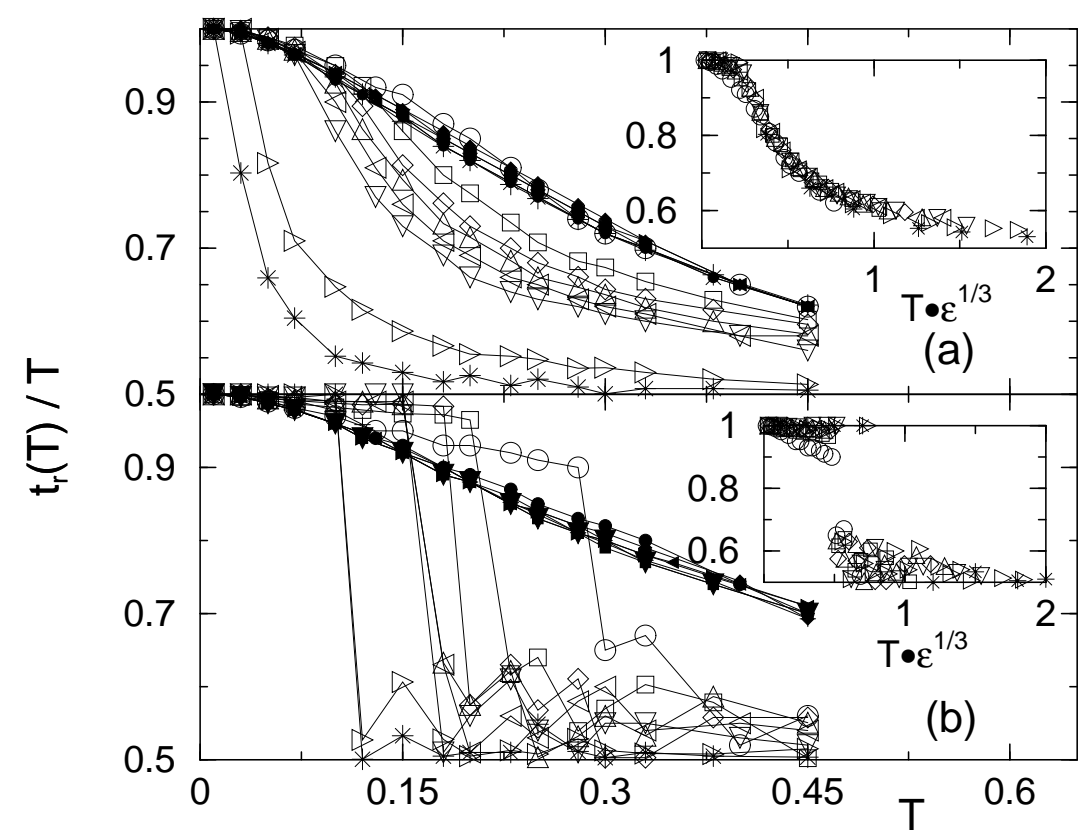

FIG.2: (a) The time $t_{r}$ calculated from $\delta E(t ; T)$, as a function of $T$ for amplitudes $\epsilon \leq 40$. The clustering of curves corresponds to $\epsilon \leq 2.5$. The inset (where only data with $\epsilon>5$ are displayed) demonstrates the scaling against $\epsilon^{1 / 3} \times T$ in the non-perturbative regime. (b) The same, but now $t_{r}$ is evaluated from $\mathcal{P}(t ; T)$.

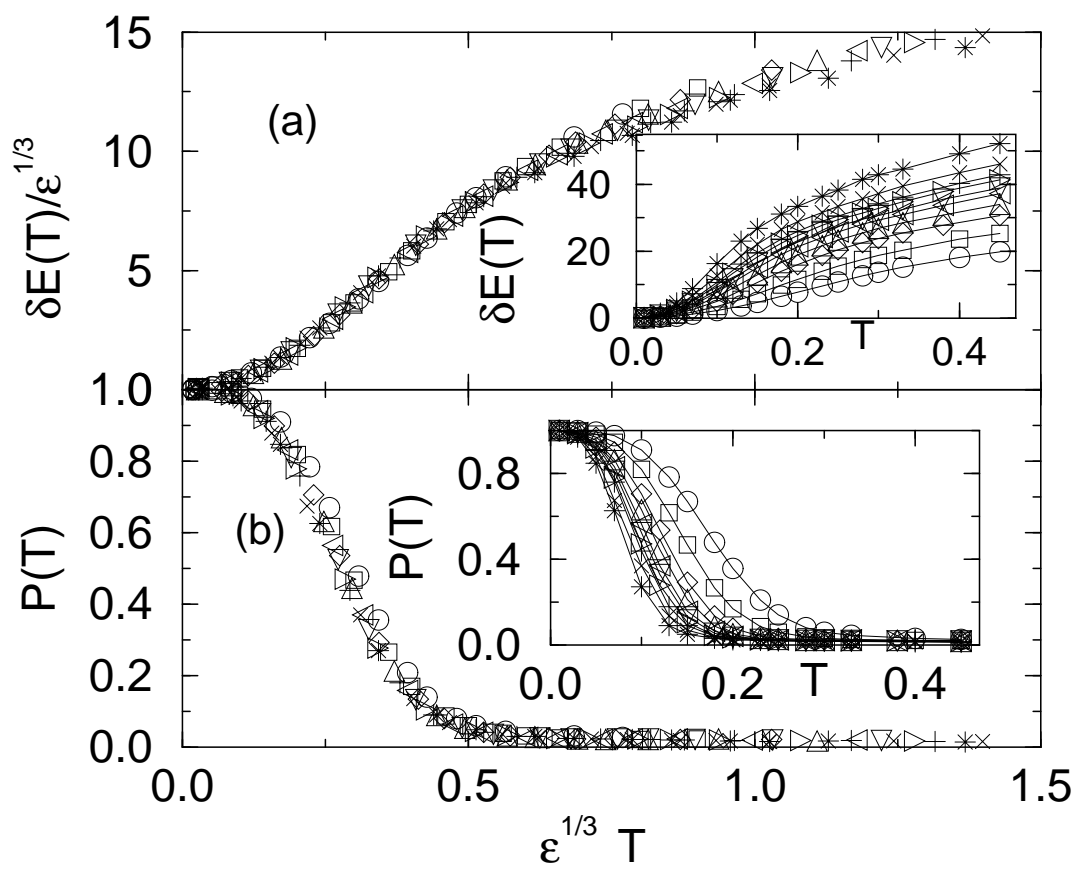

FIG.3: (a) The rescaled energy spreading $\delta E(T) / \epsilon^{1 / 3}$ versus the rescaled time $\epsilon^{1 / 3} \times T$ for $\epsilon$ values in the non-perturbative regime $(\epsilon>5)$. The inset displays the same data without rescaling. (b) The same for the survival probability $\mathcal{P}(T)$. 